\title{
On the nature of cosmological redshift and spectral shift in Schwarzschild-like and other spacetimes
}

\author{
Andrzej Radosz • Andy T. Augousti · Alicja Siwek
}

Received: 31 July 2012 / Accepted: 12 December 2012 / Published online: 25 December 2012 C) The Author(s) 2012. This article is published with open access at Springerlink.com

\begin{abstract}
The kinematical nature of the spectral shift in different types of spacetime, cosmological redshift and frequency shift in Schwarzschild spacetime being the most prominent representatives, has been a subject of recent interest. We demonstrate that the kinematical nature of the spectral shift is an inherent feature of the particular type of spacetimes. The general solution is found: necessary and sufficient conditions for the spectral shift arising from a kinematical origin are formulated. The status of the relative speed is discussed.
\end{abstract}

Keywords Generalized Doppler effect - Relative and recession speeds ·

Schwarzschild spacetime · Friedmann-Lemaître-Robertson-Walker spacetime

\section{Introduction}

Communication by means of the exchange of electromagnetic signals is accompanied by a frequency shift. This is a combined effect involving, in the general case, entangled contributions of kinematical and gravitational origins. Recently, various aspects of this problem have been discussed, displaying decoupling and in consequence, factorization or partial factorization into gravitational and kinematical (time-dilation-like and classical Doppler shift) contributions [1-3] in the case of Schwarzschild spacetime. Nevertheless, as shown long ago by Synge [4], the ratio of the frequencies, the generalized Doppler shift, measured by observer $\mathrm{O}$ and sent from source $\mathrm{S}$ may be expressed as

A. Radosz · A. Siwek $(\varangle)$

Institute of Physics, Wrocław University of Technology, Wrocław, Poland e-mail: alicja.siwek@pwr.wroc.pl

\section{A. T. Augousti}

Faculty of Science, Engineering and Computing, Kingston University,

Kingston, Surrey KT1 2EE, UK 


$$
\frac{\omega_{O}}{\omega_{S}}=\frac{1}{\left(\sqrt{1-v_{S O}^{2}}\right)^{-1}+v_{R}}
$$

where dimensionless notation here and in the rest of this paper, taking $c=1$, is used. In Eq. (1) two distinct speeds-the relative speed between the source and the observer $v_{S O}$ and the so-called recession speed $v_{R}$ (see below)—have been introduced.

Recently, the nature of the cosmological redshift has been the subject of controversy [5-7]. Narlikar [5] and then Bunne and Hogg [6] showed that cosmological redshift depends solely on relative speed; hence it is a purely kinematical effect. Chodorowski [7] argued that this is a more complex effect, involving also a gravitational contribution. In both these views the relative speed, $v_{S O}$, is defined in terms of the scalar product of O's velocity and parallel transported the velocity of $\mathrm{S}$ to $\mathrm{O}$. The difference between the claims of the authors in [5,6] and [7] appears to be due to the different paths for the parallel transport: $S^{\prime}$ velocity is shifted along a null geodesic in the former case, and is shifted on the hypersurface of constant time in the latter case. Two particular issues in this discussion are interesting. Firstly, it was shown in [5] that the frequency shift for particular class of observers in Schwarzschild spacetime, takes the same form as cosmological redshift in Friedmann-Lemaître-Robertson-Walker (FLRW) spacetime. Secondly, the speed characterizing this ratio is applied in Ref. [7] to discuss cosmological speeds; the author infers that the speeds in the expanding universe are limited by the value of the speed of light (see however [8]).

The main issue of this paper is to establish how general this effect is, namely that the spectral shift has a kinematic origin. We will discuss this question, providing what we hope will be a definitive answer. The other side of this problem concerns the meaning of the relative speed itself. Although there is no invariant definition of such a quantity, one can find a suitable background for the discussion of the status of "relative speed". In this way one could validate the statements in [7] associated with the meaning of speeds in the expanding universe.

The paper is organized as follows. In the following section we will show that the kinematical origin of the spectral shift in Schwarzschild and in FLRW spacetimes is a special case of a more general situation. The general character of this result will be the subject of Sect. 3. In Sect. 4 the status of the relative speed will be considered within a Schwarzschild spacetime. A final discussion and conclusions will be given in the last section.

\section{Kinematical origin of spectral shift: Schwarzschild spacetime and FLRW geometry}

In the general case of a source $\mathrm{S}$ and an observer $\mathrm{O}$ the ratio of the frequencies of received and emitted electromagnetic signal may be expressed as a ratio of scalar products:

$$
\frac{\omega_{O}^{r}}{\omega_{S}^{e}}=\frac{U_{O}^{\alpha} k_{O \alpha}}{U_{S}^{\alpha} k_{S \alpha}}
$$


where $U$ denotes the 4-velocity vector, $U^{\alpha} U_{\alpha}=1$, and $k$ denotes a wave vector (tangential to a null geodesic) $k^{\alpha} k_{\alpha}=0$, indices $\mathrm{r}(\mathrm{e})$ stand for receiving(emitting). The frequency shift (2) may be given in terms of two speeds, as shown in Eq. (1). It was pointed out recently [5-7] that in specific cases further simplification occurs and Eq. (2) is a function of a single parameter, namely relative speed:

$$
\frac{\omega_{O}^{r}}{\omega_{S}^{e}}=\sqrt{\frac{1 \mp v_{S O}}{1 \pm v_{S O}}} .
$$

This is referred to as the kinematical origin of a spectral shift. It was shown $[5,6]$, that Eq. (3) with the upper label applies for co-moving observers in FLRW spacetime resulting in the cosmological redshift. In the case of Schwarzschild geometry [5], for resting $\mathrm{S}$ and $\mathrm{O}$, Eq. (3) was found to reproduce the well-known gravitational red- or blueshift. However different these two spacetimes are, the result (3) turns out to be a common property and, as shown below, it holds for a wider class of observers. Having this in mind, we will discuss a more general case.

Let us consider the case of $\mathrm{S}$ and $\mathrm{O}$ exchanging signals in the $t-r$, time-radial (direction) region of a spacetime with a metric tensor block diagonal, $t-r$ sector orthogonal to the $\theta, \phi$ one. We shall assume in the $t-r$ region

$$
d s^{2}=g_{t t} d t^{2}+g_{r r} d r^{2}
$$

a special property of this spacetime, namely that the metric tensor is diagonal in this range. One can express the spectral shift (2) in terms of the velocity vector $U_{S}$ and wave vector $k_{S}$ parallel transported from $\mathrm{S}$ to $\mathrm{O}$, denoted as $U_{S / O}$ and $k_{S / O}$. Two obvious relations hold. First of them is the conserved scalar product $U_{S / O} k_{S / O}=U_{S} k_{S}$. Second, when they are parallel transported along a null geodesic, then $k_{S / O}=k_{S}$. They lead then to the spectral shift (2) given as:

$$
\frac{\omega_{O}}{\omega_{S}}=\frac{U_{O}^{t}+\lambda U_{O}^{r}}{U_{S / O}^{t}+\lambda U_{S / O}^{r}}
$$

where $\lambda= \pm \sqrt{\left|\frac{g_{r r}}{g_{t t}}\right|}$. Indeed, $k$ is a null vector, so

$$
\left(\frac{k_{O}^{r}}{k_{O}^{t}}\right)^{2}=-\frac{g_{r r}}{g_{t t}} \equiv \lambda^{2} .
$$

On the one hand, relative speed is defined as:

$$
U_{S / O}^{\alpha} U_{O \alpha} \equiv \frac{1}{\sqrt{1-v_{S O}^{2}}}
$$

and on the other hand, $U_{O}$ and $U_{S / O}$ obey, in this case, the relation: 


$$
U_{S / O}^{\alpha} U_{O \alpha}=g_{t t}\left(U_{O}^{t} U_{S / O}^{t}-\lambda^{2} U_{O}^{r} U_{S / O}^{r}\right)
$$

Utilizing Eqs. (5) and (8) one obtains:

$$
U_{S / O}^{\alpha} U_{O \alpha}=\frac{1}{2}\left(\frac{\omega_{O}}{\omega_{S}}+\frac{\omega_{S}}{\omega_{O}}\right) .
$$

Applying definition (7) one reproduces the formula (3). Therefore, the spectral shift may be given in terms of the relative speed $v_{S O}$. It turns out to be a general property of co-radial observers in the case of a spacetime with a block-diagonal metric tensor and diagonal $t-r$ sector. Schwarzschild's spacetime and FLRW spacetime with the metric tensor diagonal are important members of this type of spacetime. One can apply the above considerations to illustrate how observers other than co-moving (FLRW) and resting (Schwarzschild) might be taken into account.

Starting from Schwarzschild spacetime, we will explicitly re-derive the frequency shift for arbitrary radial motion of source and observer as being of kinematic nature.

\subsection{Schwarzschild spacetime}

The line element expressed in Schwarzschild coordinates takes the form:

$$
\begin{aligned}
d s^{2} & =\left(1-\frac{r_{S}}{r}\right) d t^{2}-\left(1-\frac{r_{S}}{r}\right)^{-1} d r^{2}-r^{2} d \theta^{2}-r^{2} \sin ^{2} \theta d \phi^{2} \\
& \equiv g_{t t} d t^{2}+g_{r r} d r^{2}+g_{\theta \theta} d \theta^{2}+g_{\phi \phi} d \phi^{2}
\end{aligned}
$$

A radial light ray, following a null geodesic, is characterized by a tangential wave vector with (conserved) time component, $k_{t}$ (see [9]) and radial component, $k_{r}$ given by

$$
k_{t}=\omega_{\infty}, \quad k_{r}= \pm \frac{\omega_{\infty}}{g_{t t}},
$$

where $\omega_{\infty}$ is the frequency parameter (measured by a distant inertial observer); \pm corresponds to "in-" and "outgoing" signal respectively. Such a signal goes radially from $\mathrm{S}$ to $\mathrm{O}$. In the case of $\mathrm{S}$ and $\mathrm{O}$, both at rest, $U^{\alpha}=\frac{1}{\sqrt{g_{t t}}} \delta_{t}^{\alpha}$, and the frequency shift,

$$
\frac{\omega_{O}}{\omega_{S}}=\sqrt{\frac{g_{t t}(S)}{g_{t t}(O)}}
$$

follows straightforwardly from Eqs. (2), (11). In the case of $\mathrm{S}$ being arranged in a stronger gravitational field, $r_{S}<r_{O}$, redshift is observed, $g_{t t}(S)<g_{t t}(O)$, and one takes the upper label, "-" in formula (3); in the opposite case, gravitational blueshift takes place corresponding to the lower label, " + " in (3). In the more general case of arbitrary radial motion of $\mathrm{S}$ and $\mathrm{O}$, frequency red- or blueshift would be described by Eq. (3) with the upper or lower label, respectively. 


\subsection{FLRW spacetime}

In this case the line element is:

$$
d s^{2}=d t^{2}-a^{2}(t)\left[\frac{d r^{2}}{1-k r^{2}}+r^{2} d \theta^{2}+r^{2} \sin ^{2} \theta d \phi^{2}\right]
$$

A radial null geodesic is characterized by wave vector

$$
k_{t}=\frac{A}{a(t)} k_{r}= \pm \frac{A}{\sqrt{1-k r^{2}}} .
$$

The frequency shift (2) as measured by two co-moving cosmic observers $\mathrm{S}$ and $\mathrm{O}$, with $U^{\alpha}=\delta_{t}^{\alpha}$, becomes an (almost) trivial calculation (see however [5]!),

$$
\frac{\omega_{O}}{\omega_{S}}=\frac{a\left(t_{S}\right)}{a\left(t_{O}\right)} .
$$

This is the cosmological redshift and it is expressed by Eq. (3), with an obvious choice of an upper label. In the more general case of an arbitrary radial motion of source and observer, the frequency might in principle be red- or blueshifted, with upper or lower label in Eq. (3), respectively.

\section{The basis of the kinematical origin of spectral shift}

The spectral shift is given by Eq. (3) for a class of observers and for the spacetimes of different properties, satisfying the block-diagonal condition for the metric tensor. But this is not the most general solution for the question of the kinematical origin of the spectral shift. The solution is the following.

Let us consider the arrangement of the three vectors: $U_{O}, k_{O}$ and $U_{S / O}$. Vectors $U_{O}$ and $k_{O}$ constitute the plane, which will be denoted as $\Pi_{O}$. If $U_{S / O}$ belongs to that plane, then the frequency shift is expressed in terms of the relative speed and the effect may be referred to as being of kinematical origin. If $U_{S / O}$ does not belong to this plane the frequency shift cannot be expressed in terms of the relative speed (only).

To prove this a unit spatial vector, $V_{O}$, belonging to the plane $\Pi_{O}$, orthogonal to $U_{O}$,

$$
V_{O}^{2}=-1, \quad V_{O} \cdot U_{O}=0
$$

is introduced. One can choose its direction in such a way that $k_{O} \cdot V_{O}=k_{O} \cdot U_{O}$. If $U_{S / O}$ also belongs to $\Pi_{O}$, then it is decomposed as follows:

$$
U_{S / O}=\alpha U_{O}+\beta V_{O}
$$


where the two components satisfy $\alpha^{2}-\beta^{2}=1$. In this case, the scalar product of $U_{S / O}$ and wave vector,

$$
U_{S / O} \cdot k_{O}=\alpha U_{O} \cdot k_{O}+\beta V_{O} \cdot k_{O}=(\alpha+\beta) U_{O} k_{O}
$$

leads to the already known formula, (9)

$$
\alpha=U_{S / O} \cdot U_{O}=\frac{1}{2}\left(\frac{\omega_{O}}{\omega_{S}}+\frac{\omega_{S}}{\omega_{O}}\right) .
$$

This result reproduces Eq. (3). So if the parallel transported velocity vector is co-planar with the velocity vector and wave vector at the destination point $\mathrm{O}$ then the frequency shift is expressed in terms of the relative speed. Let us also note that in this case $\beta$ is Synge's "recession speed", Eq. (1), $v_{R}=\beta$. The rest of the proof is obvious and we will skip it.

In this context one can make a more general comment about the kinematical origin of the frequency shift in particular cases. The spacetimes identified in the previous section, with FLRW and Schwarzschild spacetimes as the important representatives, satisfy the condition of co-planar arrangement of $U_{O}, k_{O}$ and $U_{S / O}$ in the case of arbitrary co-radial motion of $\mathrm{S}$ and $\mathrm{O}$.

The case of Kerr spacetime is interesting due to its axial symmetry and the nondiagonal character of its metric tensor,

$$
\begin{aligned}
d s^{2}= & g_{t t} d t^{2}+2 g_{\phi t} d \phi d t+g_{r r} d r^{2}+g_{\theta \theta} d \theta^{2}+g_{\phi \phi} d \phi^{2} \\
= & \left(1-\frac{2 M r}{\rho^{2}}\right) d t^{2}+\frac{4 M r a \sin ^{2} \theta}{\rho^{2}} d \phi d t-\frac{\rho^{2}}{\Delta} d r^{2} \\
& -\rho^{2} d \theta^{2}-\left[\left(r^{2}+a^{2}\right) \sin ^{2} \theta+\frac{2 M r a^{2} \sin ^{2} \theta}{\rho^{2}}\right] d \phi^{2},
\end{aligned}
$$

where $\rho^{2}=r^{2}+a^{2} \cos ^{2} \theta, \Delta=r^{2}+a^{2}-2 M r$.

The condition $U_{S / O} \in \Pi_{O}$ is satisfied in a more or less obvious case. It is the case of a signal travelling along the symmetry axis, $\theta=0$. On this axis the metric trivializes to the radial Schwarzschild-like metric. For arbitrary $\mathrm{S}$ and $\mathrm{O}$ motion along the symmetry axis the condition of co-planar arrangement of the three vectors mentioned above is satisfied: one reproduces the kinematic origin of the frequency shift.

The non-trivial case is the situation of exchange of signals between static observers in the equatorial plane (above the ergo-sphere). In this case, due to an (energy) conservation law, one finds the frequency shift:

$$
\frac{\omega_{O}}{\omega_{S}}=\sqrt{\frac{g_{t t}(S)}{g_{t t}(O)}}
$$

preserving the same form as in Schwarzschild spacetime. Co-radial arrangement of S and $\mathrm{O}$ in Schwarzschild spacetime would result, as already argued, in the kinematical 
origin interpretation. This is not the case in Kerr spacetime where O and S, Eq. (21), are not even co-radial. Hence, the frequency shift is not of a kinematic origin in this case even for resting $\mathrm{S}$ and $\mathrm{O}$. However, at location $\mathrm{O}$ one can find an observer $\mathrm{O}^{\prime}$ whose relative speed with respect to $\mathrm{S}, v_{S O^{\prime}}^{2}=v_{S O}^{2}$ and for whom the frequency shift is of kinematical origin (3). This is done determining $U_{S / O}, k_{O}$ plane and spatial unit vector $V_{S / O}$, belonging to that plane and orthogonal to $U_{S / O}$,

$$
V_{S / O}^{2}=-1, \quad V_{S / O} \cdot U_{S / O}=0
$$

Then one can find such an observer $\mathrm{O}^{\prime}$ (with velocity $U_{O^{\prime}}$ ), whose speed relative to $\mathrm{S}$ would be $v_{S / O}$ :

$$
U_{S / O} \cdot U_{O^{\prime}}=U_{S / O} \cdot U_{O} \equiv \frac{1}{\sqrt{1-v_{S O}^{2}}}
$$

Indeed, $U_{O^{\prime}}$ is the following (unit) vector

$$
U_{O^{\prime}}=\mu U_{S / O}+v V_{S / O}
$$

where, $\mu=U_{O} \cdot U_{S / O}, v=\sqrt{\mu^{2}-1}$ and consequently the relative speeds of $\mathrm{O}^{\prime}$ and $\mathrm{O}$ versus $\mathrm{S}$ are the same and the frequency shift

$$
\frac{\omega_{O^{\prime}}}{\omega_{S}}=\sqrt{\frac{1 \pm v_{S O}}{1 \mp v_{S O}}}
$$

is of kinematical origin. This is a general procedure and might be applied for arbitrary observers in Kerr spacetime and also in other spacetimes. One of its consequences is the fact that the spectral shift for such an $\mathrm{O}^{\prime}$, Eq. (24), is the largest possible, i.e. $\frac{\omega_{O^{\prime}}}{\omega_{S}} \geq \frac{\omega_{O}}{\omega_{S}} \geq 1$ or $\frac{\omega_{O^{\prime}}}{\omega_{S}} \leq \frac{\omega_{O}}{\omega_{S}} \leq 1$.

\section{The status of the relative speed (in Schwarzschild spacetime)}

Relative speed is given in terms of velocity parallel transported along null geodesics. Chodorowski [7] suggested that in FLRW spacetime parallel transport should be taken on the hypersurface of the constant cosmic time with the resulting speed, referred to as "recession speed". This quantity was then utilized in cosmology leading to the final conclusion that speeds in the expanding universe could not be superluminal. Relative speed or recession speed is not an invariant quantity, as depending on the path of the parallel transport so any ultimate statement concerning the status of each of them as a meaningful tool describing a kinematical component of the spectral shift can hardly be made. Indeed, there is concern regarding its physical sense (e.g. see [10]). We will apply the relative speed in the case of Schwarzschild spacetime where there are no doubts concerning the kinematical component of spectral shift and local speed is a 
well defined quantity. In these circumstances the status of the relative speed will be discussed.

We shall consider two co-radial observers in Schwarzschild spacetime, in this section denoted as $\mathrm{A}$ and $\mathrm{B}$. The relation between frequency $\omega_{A}$ and A's speed $v_{A}$ as measured by a local resting observer $\mathrm{R}$, is [3]:

$$
\omega_{A}=\omega_{\infty} \frac{1 \mp v_{A}}{\sqrt{g_{A}} \sqrt{1-v_{A}^{2}}}
$$

where $\mp$ labels the parallel/antiparallel directions of $v_{A}$ and the light ray. In this way kinematical and gravitational components factorize [3]. The relative speed may be determined via frequency shift, by means of the scalar product (7).

Using these tools relative speed may be related to directly measurable quantities in some specific cases:

(a) Static observers, $A\left(r_{A}\right)$, and $B\left(r_{B}\right)$

Local speeds vanish, $v_{B}=v_{A}=0$ and the nature of the frequency shift is purely gravitational, (blue- or redshift)

$$
\frac{\omega_{B}^{r}}{\omega_{A}^{e}} \equiv f(A \rightarrow B)=\frac{\sqrt{g_{A}}}{\sqrt{g_{B}}}=f^{-1}(B \rightarrow A) \equiv\left(\frac{\omega_{A}^{r}}{\omega_{B}^{e}}\right)^{-1}
$$

Relative speed B versus A does not vanish and is given in terms of the strength of the gravitational field

$$
v_{B / A}^{2}=\left(\frac{g_{A}-g_{B}}{g_{A}+g_{B}}\right)^{2} \equiv v_{A / B}^{2} .
$$

This is a special case when values of relative speeds, $v_{B / A}$ and $v_{A / B}$ are equal.

(b) Static $B\left(r_{B}\right)$ and A infalling from the starting point $r_{B}$

In this case $v_{B}=0, v_{A} \neq 0$; simple calculation shows (see [3]) that the frequency shift turns out to be purely kinematical (the classical Doppler effect), see also [11]

$$
\frac{\omega_{A}^{r}}{\omega_{A}^{e}} \equiv f(B \rightarrow A)=\frac{1}{1+v_{A}} .
$$

Relative speed $v_{B / A}^{2}$ is expressed via $v_{A}$

$$
v_{B / A}^{2}=\left(\frac{1-\left(1+v_{A}\right)^{2}}{1+\left(1+v_{A}\right)^{2}}\right)^{2}
$$

There is no symmetry between signals B to A and A to B: $v_{A} \rightarrow-v_{A}$.

Two outcomes are worth emphasizing. The relative speed does not vanish even in the case of both $\mathrm{A}$ and $\mathrm{B}$, being at the rest (27). Its value may come close to the 
value of the speed of light when the location of one of the observers approaches the event horizon. A well-known feature of the Schwarzschild spacetime [11] is that when A approaches the event horizon, its speed tends to the speed of light, $v_{A} \rightarrow 1$. Then,

$$
v_{B / A}^{2} \rightarrow\left(\frac{3}{5}\right)^{2}, \text { and } v_{A / B}^{2} \rightarrow 1
$$

Observer A, penetrating the range beneath the event horizon, would still receive signals emitted above the horizon and the definition of relative speed of source and observer may still be utilized. Below the horizon, applying Kruskal coordinates [12], one can extend formula (28), finding:

$$
v_{B / A}^{2}=1-4\left[\frac{1+x_{A}}{1+\left(1+x_{A}\right)^{2}}\right]^{2}\left\{\begin{array}{l}
\stackrel{r \rightarrow r_{S}}{\longrightarrow}\left(\frac{3}{5}\right)^{2} \\
\underset{r \rightarrow 0}{\longrightarrow} 1
\end{array} \quad \text { where } x_{A}=\frac{\sqrt{\frac{2 M}{r_{A}}-\frac{2 M}{r_{B}}}}{\sqrt{1-\frac{2 M}{r_{B}}}} \underset{r<2 M}{>} 1\right.
$$

The relative speed $v_{B / A} \rightarrow 1$, for resting $\mathrm{B}$ and for A reaching the singularity.

\section{Discussion and concluding remarks}

In general the spectral shift may be expressed in terms of two parameters, the relative speed and another one, a quantity, referred to by Synge as a recession speed. Both of them are defined via the scalar product of velocity vector of an "observer" $O$ and parallel transported "source" $S^{\prime}$ velocity. Recent interests were associated with the particular case of a spectral shift being represented solely by relative speed. In this situation, found in particular cases, the cosmological redshift being the most prominent representative, the spectral shift was referred to as of kinematical origin. We have studied here the nature of this property, namely, the spectral shift (or a generalized Doppler effect) being of kinematical origin i.e. being expressed in terms of the relative speed. It was shown that if at O's location, three vectors, $U_{O}, k_{O}$ and $U_{S / O}$ are co-planar then the frequency shift is a function of relative speed and takes the form (3). It was found that co-radial observers in spacetimes with blockdiagonal metric (diagonal in $t-r$ range) satisfy this condition. The metric tensors in Schwarzschild and Schwarzschild-like spacetimes in Hor̂av-Lifshitz gravity (see also [13]) and FLRW spacetime are diagonal, so in these cases the spectral shift may reveal a kinematic origin. This conclusion does not apply to Kerr spacetime though there are some specific groups of observers recording a kinematic character of the frequency shift. As discussed very recently [14], the Doppler shift derived both in terms of light rays and beyond this approximation, does not appear to reveal either a kinematical origin, or is expressed in terms of local speed(s). However, even in this case, one would be able to reconstruct the arrangement of observers reproducing a frequency shift as being of kinematic origin. The construction proposed in this context turns out to be applicable in an arbitrary case. 
We have thrown light on the interpretation and status of relative speed, discussing the case of Schwarzschild spacetime. It is well-known that the frequency shift for two resting observers is a purely gravitational effect and that for a freely falling observer receiving signals from its "mother station" it is a purely kinematic effect. When expressed in terms of relative speed, the frequency shift takes the form (3), apparently leading to the conclusion about its kinematical character in both cases. In the case of resting observers, relative speed turned out to be a non-vanishing quantity. It was found to tend to a peculiar limit 3/5, where the local speed approached 1 .

On the basis of this discussion one can hardly regard relative speed as a characteristic of the kinematic component of the spectral shift. Can one apply a similar tool for interpreting speeds in an expanding universe? Chodorowski [7] argued that in FLRW cosmology a proper way of discussing the kinematic component of the cosmological redshift was "recession speed". Not disregarding this tool we would like to underline one of the main issues formulated in Ref. [7]: "definition (in Ref. [6]) and ours imply the same very important conclusion: recession velocities (...) are subluminal”. Let us emphasize then, that both "recession speed" as well as relative speed are by definition subluminal. As shown above, yet in Schwarzschild spacetime relative speed, in the extreme case of an observer approaching the black hole's ultimate singularity, whose speed appears to tend to infinity [in a sense of an above-event-horizon frequency shift $1 /\left(1+v_{A}\right)$ approaching zero (see also $\left.\left.[13,15]\right)\right]$, tends to 1 .

The final conclusion is the following. A kinematical description (i.e. a description in terms of relative speed) of the spectral shift is artificial once one goes beyond a narrow class of solutions and observers (namely co-radial observers in spacetimes with a block-diagonal metric, which are also diagonal in the $t-r$ range).

Acknowledgments The authors would like to thank the anonymous reviewer of this manuscript for critical remarks and subtle suggestions affecting the outcomes of this paper in an unexpectedly substantial manner.

Open Access This article is distributed under the terms of the Creative Commons Attribution License which permits any use, distribution, and reproduction in any medium, provided the original author(s) and the source are credited.

\section{References}

1. Bahder, T.B.: Relativity of GPS measurement. Phys. Rev. D 68, 063005 (2003)

2. Linet, B., Teyssandier, P.: Time transfer and frequency shift to the order $1 / c^{4}$ in the field of an axisymmetric rotating body. Phys. Rev. D 66, 024045 (2002)

3. Radosz, A., Augousti, A.T., Ostasiewicz, K.: The Doppler shift in a Schwarzschild spacetime. Phys. Lett. A 373, 801-803 (2009)

4. Synge, J.L.: Relativity: The General Theory. North-Holland Publishing Company, Amsterdam (1960)

5. Narlikar, J.V.: Spectral shifts in general relativity. Am. J. Phys. 62, 903 (1994)

6. Bunn, E.F., Hogg, D.W.: The kinematic origin of the cosmological redshift. Am. J. Phys. 77, 688 (2009)

7. Chodorowski, M.: The kinematic component of the cosmological redshift. Mon. Not. R. Astron. Soc. 413, 585-594 (2011)

8. Tegmark, M.: The multiverse hierarchy. In: Carr, B. (ed.) Universe or Multiverse? pp. 99-126. Cambridge University Press, Cambridge (2007) (arXiv:0905.1283)

9. Okun, L.B., Selivanov, K.G., Telegdi, V.L.: On the interpretation of the redshift in a static gravitational field. Am. J. Phys. 68, 115 (2000) 
10. Bolos, V.J.: A note on the computation of geometrically defined relative velocities. Gen. Relativ. Gravit. 44, 391-400 (2012)

11. Radosz, A., Augousti, A.T., Ostasiewicz, K.: A useful expression for relativistic energy conservation of a point mass in an isotropic static gravitational field. Eur. J. Phys. 32, 535 (2011)

12. Gawelczyk, M., Polonyi, J., Radosz, A., Siwek, A.: Exchange of signals around the event horizon in Schwarzschild space-time (under review) (arXiv:1201.4250)

13. Radosz, A., Siwek, A., Polonyi, J., Ostasiewicz, K.: Circular geodesics in Schwarzschild-like spacetimes. Mod. Phys. Lett. A 26, 473-479 (2011)

14. Cisneros, S., Goedecke, G., Beetle, C., Engelhardt, M.: On the Doppler effect for light from orbiting source in Kerr-type metrics (arXiv:1203.2502)

15. Popławski, N.J.: Radial motion into an Einstein-Rosen bridge. Phys. Lett. B 687, 110 (2010) 